\section{A Light Source for locating Cstrogens on Chromatograms}

As part of an investigation at this Station into the reproductive physiology of sheep, a suitable light source was required for detecting traces of cestrogens on paper chromatograms of blood extracts. GEstrogens have an absorption maximum near $280 \mathrm{~m} \mu$, and light near this wave-length gives maximum contrast when used for photographing them on filter paper. The mercury-vapour lamp is commonly used for this purpose, but its emission in this region is weak and a train of filters is required.

On the other hand, the magnesium spark emits most of its photographically active radiation between 2791 and $2803 \mathrm{~A}$., and a spark system assembled from readily available components has proved simple and convenient in operation. The transformer was of a type used commercially for neon lighting, with a secondary rating of $15,000 \mathrm{~V} ., 30 \mathrm{~m}$.amp., the $0.0025 \mu \mathrm{F}$. condenser was assembled by soldering forty $0.1 \mu \mathrm{F}$., $500 \mathrm{~V}$. radio condensers in series and the $0.01 \mu \mathrm{H}$. coil consisted of seven turns of 14-gauge copper wire on a 3 -in. diam. 'Tufnol' tube. The clectrodes were made by winding two helices of magnesium ribbon about $1 \mathrm{~cm}$. in diameter held approximately $6 \mathrm{~mm}$. apart.

Photographs taken through No. 1 Whatman filter paper in contact with Ilford reflex document paper using 10 sec. exposure at $70 \mathrm{~cm}$. enabled spots down to $1 \mu \mathrm{gm}$. œestrone/sq. cm. to be detected. Using a 4-cm. filter of nickel sulphide and cobalt sulphate to reduce undesirable radiation failed to give any apparent increase in sensitivity.

Although the spark system is run in air, the electrodes can be operated continuously for periods up to one minute without igniting.

$$
\text { D. D. Perrin }
$$

Ruakura Animal Research Station, Hamilton, New Zealand. Sept. 20.

\section{A New Detector for Vapour Phase Chromatography}

VAPOUR phase chromatography has been used with great success in these laboratories for the analysis of the products of reactions in the gas phase. By using a thermal conductivity detector it has been possible to estimate amounts of product of the order of $10^{-8}$ mole; but this was the lowest limit which could be reached. In certain cases, however, it became neces. sary to detect $10^{-12}$ mole of a substance. The detector described below has been developed to meet this

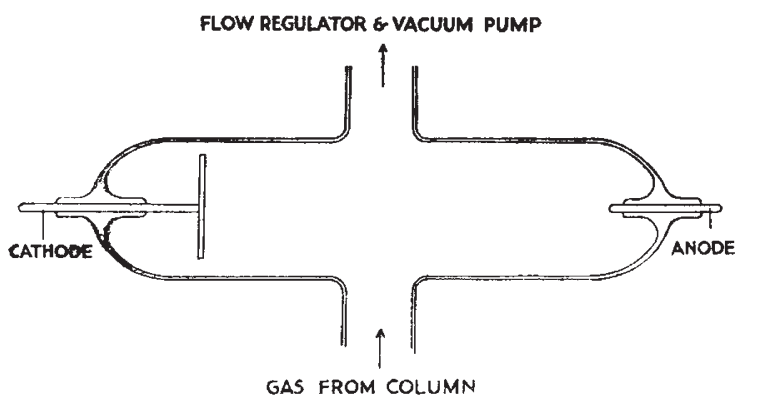

Fig. 1. Glow-discharge detector

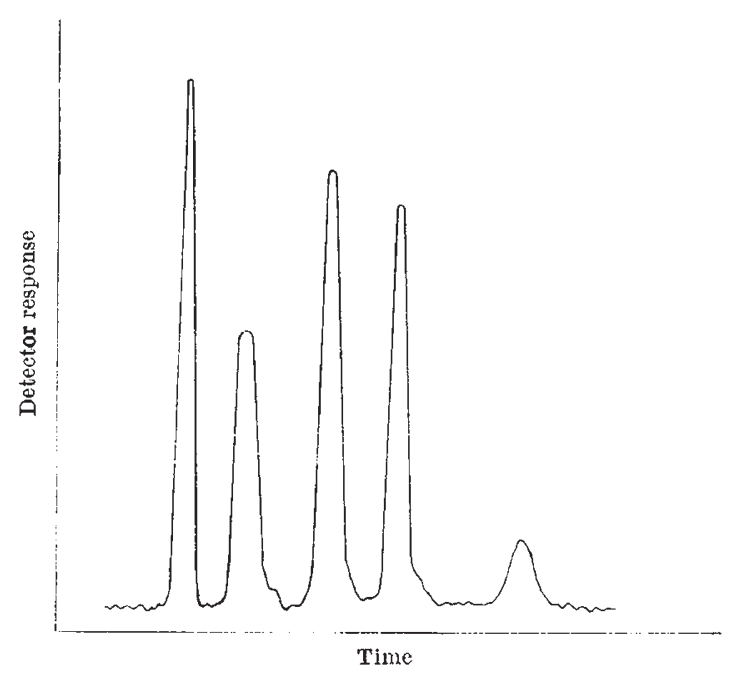

Fig. 2. Detection of components of paraffn mixture

requirement. It is of wide applicability and is easily constructed.

Use is made of the fact that the voltage existing across a normal gaseous discharge is a function of the type of gas used. This voltage changes by several volts with the introduction of a minute amount of impurity into the gas. The detector consists of a small platinum disk cathode and a tungsten wire anode (Fig. 1) forming one arna of a Wheatstone bridge which is supplied with 900 volts from a stabilized d.c. source. The bridge is balanced with carrier gas flowing through the detector at a pressure sufficiently low to produce a normal glow discharge between the electrodes. Compounds eluted from the column cause a voltage change across the detector which is measured by a recording potentiometer. Fig. 2 shows a recording of the constituents separated from $10^{-10} \mathrm{~mole}$ of petroleum ether $\left(40-60^{\circ}\right)$ on a 4 -ft. column of 'Celite' and amyl phthalate. Details of the technique will be published later.

\section{J. HaRley}

V. Pretorrus

Department of Physical Chemistry, University of Pretoria.

\section{Definition of Chromatography}

TuE essence of the classical chromatographic method of Tswett ${ }^{1}$ is presented by Ratchinsky ${ }^{2}$ in the form of the following definition: "Any liquid or gaseous mixture of the substances is divided into its components during the process of its movement through a layer of sorbent, if there are differences in sorbtion interaction between the components of the mixture and the sorbent". I believe the relative movement of the phases to be the important feature; in the case of the movement of the dispersed sorbent through a layer of a liquid or gaseous mixture the chromatographic process of Tswett will be inverted. In relation to the aggregate state of the dispersed sorbent the inverted method of 'Tswett thus embraces three varieties of chromatography : (1) suspension chromatography-when the solid dispersed particles of the sorbent settle or rise through the liquid or gaseous mixture ${ }^{3.4}$; (2) emulsion chromatography when the dispersed drops of liquid move through the 